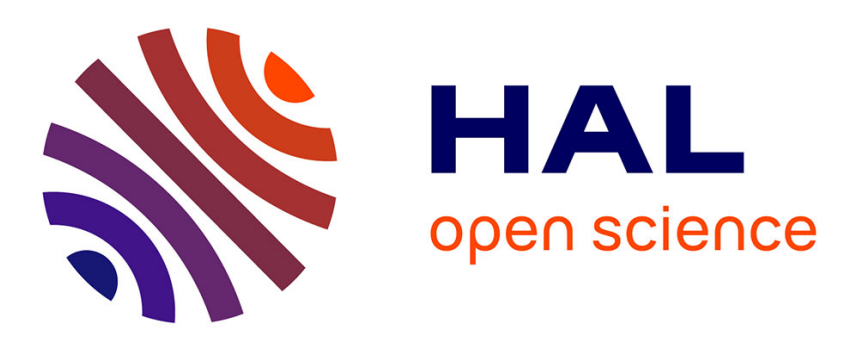

\title{
Calculation of $\mathrm{Zn}, \mathrm{Cd}, \mathrm{Hg}$ adsorption on graphene with incremental CCSD $(\mathrm{T})$ and range-separated hybrid DFT*
}

Fabian Weber, Carsten Müller, Beate Paulus, Peter Reinhardt

\section{To cite this version:}

Fabian Weber, Carsten Müller, Beate Paulus, Peter Reinhardt. Calculation of Zn, Cd, Hg adsorption on graphene with incremental $\operatorname{CCSD}(\mathrm{T})$ and range-separated hybrid DFT*. Molecular Physics, 2016, 114 (7-8), pp.1098-1109. 10.1080/00268976.2015.1137644 . hal-01323725

\section{HAL Id: hal-01323725 \\ https://hal.sorbonne-universite.fr/hal-01323725}

Submitted on 31 May 2016

HAL is a multi-disciplinary open access archive for the deposit and dissemination of scientific research documents, whether they are published or not. The documents may come from teaching and research institutions in France or abroad, or from public or private research centers.
L'archive ouverte pluridisciplinaire HAL, est destinée au dépôt et à la diffusion de documents scientifiques de niveau recherche, publiés ou non, émanant des établissements d'enseignement et de recherche français ou étrangers, des laboratoires publics ou privés. 


\title{
Calculation of $\mathrm{Zn}, \mathrm{Cd}, \mathrm{Hg}$ adsorption on graphene with incremental CCSD(T) and range-separated hybrid DFT
}

\author{
Fabian Weber ${ }^{1,2}$, Carsten Müller ${ }^{1}$, Beate Paulus ${ }^{1}$, Peter Reinhardt ${ }^{2 b}$ \\ 1 Institut für Chemie und Biochemie, Freie Universität Berlin, \\ Takustraße 3, D - 14195 Berlin, Germany \\ 2 Sorbonne Universités, Laboratoire de Chimie Théorique, Université Pierre et Marie Curie, \\ 4 place Jussieu, F- 75252 Paris, France and CNRS, \\ UMR7616, Laboratoire de Chimie Théorique, \\ 4 place Jussieu, F - 75252 Paris, France
}

At the hand of the adsorption of the metal atoms $\mathrm{Zn}, \mathrm{Cd}$ and $\mathrm{Hg}$ on a graphene sheet we propose a combination of range-separated hybrid density-functional theory in combination with the incremental scheme in localized orbitals and extrapolation procedures for the description of this type of extended systems. Using only dispersion terms for the long-range part, we were able to obtain results comparable to incremental $\operatorname{CCSD}(\mathrm{T})$ calculations. Repulsive three-center increments reduce the overall correlation contribution to the binding energy by $20 \%$.

May 26, 2016

\footnotetext{
a Part of the special issue honoring the work of Andreas Savin.

b email: Peter.Reinhardt@upmc.fr; FAX: +33144274117
} 


\section{INTRODUCTION}

Range-separated density-functional theory (RSH-DFT) has been introduced in the literature by Andreas Savin in $1996,{ }^{1}$ and has been further developed in recent years, leading to a powerful tool to describe van-der-Waals interactions in a DFT framework. The long-range correlation part, based on an expansion in contributions from excited determinants, remains nevertheless a challenging task, as the advantageous scaling of DFT with system size is partly lost. On the other hand, the significant reduction of the basis-set superposition error and the possibility to use smaller basis sets than in "standard", wavefunction-based post-Hartree-Fock calculations render the combination of DFT and long-range correlation very appealing.

For a correlation treatment, localized molecular orbitals have the advantage that they both provide for an a priori fragmentation of several interacting systems, and allow for separating excitations of the long-range correlation part into intra-molecular and inter-molecular ones, depending on the site or the fragment the occupied and virtual orbitals are located on. Back in 1992 Stoll proposed $^{2-4}$ to determine the correlation energy of an extended system not from one single calculation, but from increments, i.e. contributions calculated for small model systems with only a few localized orbitals.

In the present study, all the before-mentioned aspects (range-separated DFT, orbital localization, fragments and the method of increments) are combined and applied to the adsorption of a metal atom $(\mathrm{Zn}, \mathrm{Cd}, \mathrm{Hg})$ on a graphene sheet. These systems present one of the most difficult challenges of today's quantum chemistry, as we deal neither with a molecular nor a perfectly infinite system. The solution is an asymptotic process, either diluting a periodic set-up - this has been done abundantly for different metal atoms on graphene by DFT approaches ${ }^{5-9}$ or model Hamiltonians ${ }^{10}$ - or by augmenting stepwise the dimension of the support until convergence or until arriving at a systematic extrapolation scheme. ${ }^{11}$ Since graphene is conducting, difficulties for the convergence of perturbative methods may be encountered as well. ${ }^{12}$ Very recently, Illas et al. published a systematic study of the adsorption of metal atoms on graphene within a periodic approach, using DFT with empirical van-der-Waals corrections. ${ }^{13}$ References therein provide a good literature survey of metal adsorption on a graphene sheet.

The organization of the article is as follows: after a brief review of the employed theory and the computational approach we present our results: first for global calculations, next for incremental calculations using localized orbitals, before extrapolating to an adsorption on the infinite graphene sheet and drawing our conclusions. All purely computational details as well as additional data are 
collected in supplementary material to this article.

\section{THEORY}

In the present work we need three basic ingredients: the range-separated hybrid densityfunctional theory, the random phase approximation (RPA) for the long-range correlation energy, and the method of increments for exploiting localized orbitals.

\section{A. RSHDFT}

Density functional theory in the Kohn-Sham formalism was developed and systematically applied in theoretical chemistry since the 1990 's. ${ }^{14,15}$ In following years it became the most powerful method for precise routine calculations in many domains. Nevertheless, as density, simply said, is absent between two distant fragments of a system interacting exclusively via long-range electron dispersion, the distance-dependence of the interaction energy cannot be described accurately by DFT with usual approximations (LDA, GGA). To cure this shortcoming, several propositions have been made in the past decades. The most popular are the different versions of empirical Grimme dispersion correction for common DFT functionals. ${ }^{16-18}$

Studying degeneracy in the context of DFT, Savin proposed a continuous, seamless transition procedure from Kohn-Sham DFT to multi-determinant wavefunction theory ${ }^{1}$ by separating the electron-electron interaction into a short-range and a long-range part. The former is calculated by DFT, and the latter is added through long-range exact-exchange and correlation contributions. The separation is not a geometrical one as in QM/MM methods, which define near-field and farfield regions in space around a center of interest and struggle with boundary effects, but a more fundamental one by picking out two electrons and distinguishing situations where they are close or where they are distant. Kohn et al showed ${ }^{19}$ that indeed the separation of the Coulomb interaction into a short-range DFT part and a long-range correlation part leads to a formalism, from which the van-der-Waals interaction can be extracted. We should add that the range-separation has been further extended by several other groups, leading to different classes of modern functionals. ${ }^{20-24}$

In practice the Coulomb electron-electron interaction $1 / r$ is split by help of the error function $\operatorname{erf}(x)=\frac{2}{\sqrt{\pi}} \int_{0}^{x} e^{-t^{2}} d t$ and a single parameter $\mu$ (which is fixed in the present work to the commonly accepted value of $\mu=0.5$ a.u.) into

$$
\frac{1}{r}=\frac{\operatorname{erf}(\mu r)}{r}+\frac{1-\operatorname{erf}(\mu r)}{r}
$$


and the Kohn-Sham RSH procedure is written as a minimization of an energy functional with respect to one determinant as

$$
E^{\mathrm{RSH}}=\min _{\Phi}\left\{\left\langle\Phi\left|T+V_{\mathrm{ne}}+W_{\mathrm{ee}}^{\mathrm{lr}}\right| \Phi\right\rangle+E_{\mathrm{Hxc}}^{\mathrm{sr}}\left[n_{\Phi}\right]\right\}
$$

with an explicit short-range Hartree-exchange-correlation functional, ${ }^{25} E_{\mathrm{Hxc}}^{\mathrm{sr}}$, and the long-range electron-electron interaction, $W_{\mathrm{ee}}^{\mathrm{lr}}$. The Hartree part of the functional employs the short-range part of the $1 / r$ interaction, and the long-range interaction the complementary long-range part of $1 / r$.

Carrying out the minimization leads to the equations

$$
\left(T+V_{\mathrm{ne}}+V_{\mathrm{H}}+V_{\mathrm{x}}^{\mathrm{lr}}+V_{\mathrm{xc}}^{\mathrm{sr}}\right)\left|\phi_{i}^{\mathrm{RSH}}\right\rangle=\epsilon_{i}\left|\phi_{i}^{\mathrm{RSH}}\right\rangle
$$

For arriving at the correct, and in principle exact, total energy, multi-determinant contributions for the long-range correlation part have to be added, either by minimizing $E$ with respect to a real multi-determinant wavefunction $\Psi$ through

$$
E^{\text {exact }}=\min _{\Psi}\left\{\left\langle\Psi\left|T+V_{\mathrm{ne}}+W_{\mathrm{ee}}^{\mathrm{lr}}\right| \Psi\right\rangle+E_{\mathrm{Hxc}}^{\mathrm{sr}}\left[n_{\Psi}\right]\right\} \quad,
$$

or by adding a long-range correlation part in a second step as $E_{\text {total }}=E_{\mathrm{RSH}}+E_{\mathrm{corr}}^{\mathrm{lr}}$, making use of the previously obtained RSH orbitals and orbital energies (or RSH matrix elements, equivalent to a Kohn-Sham or Fock matrix as result of the minimization of a single-determinant wavefunction). The molecular orbitals need not to be canonical orbitals, diagonalizing the RSH matrix, but may as well be localized ones. This will be expoited in the next sections. ${ }^{26}$

For instance a $\mu$-dependent long-range MP2 contribution $^{28}$ is obtained in canonical orbitals as

$$
E_{\mu, \mathrm{MP} 2}^{(2)}=\sum_{i j a b} \frac{(i a \mid j b)_{l r, \mu}\left(2(i a \mid j b)_{l r, \mu}-(i b \mid j a)_{l r, \mu}\right)}{\epsilon_{i}+\epsilon_{j}-\epsilon_{a}-\epsilon_{b}}
$$

and equivalently, if localized orbitals are used, by minimizing the Hylleraas functional, ${ }^{29}$ leading to a CEPA-like system of linear equations in the coefficients of the excited determinants. Other long-range correlation treatments may be employed like configuration interaction in size-consistent variants, coupled-cluster schemes, or the random-phase approximation, RPA.

For intermolecular interaction energies in particular, the explicit choice of the short-range functional has little influence on calculated values. Other advantages of a combination of rangeseparated DFT with a long-range correlation part are the reduced dependence on basis sets, and thus a generally small basis-set superposition error, and an advantageous scaling of the Kohn-Sham step with system size. 


\section{B. RPA}

For adding the long-range correlation to the RSHDFT energy, we use here the Random-Phase Approximation, a method which recently became popular due to its reduced needs of computational resources (reduced set of bielectronic integrals over molecular orbitals), , nevertheless more accurate than second-order perturbation theory due to self-consistently screened or collective excitations. ${ }^{30}$ The underlying RPA equations may be derived in several ways, based on single excitations and their coupling, showing thus the close connection to time-dependent DFT approaches to single-electronexcited states. ${ }^{31-33}$ Based on the different derivations, ${ }^{34}$ one may arrive at different flavors of the Random Phase Approximation, using anti-symmetrization or not for the occurring kernel, and contracting the obtained amplitudes with direct or with anti-symmetrized integrals. We employ in the following the RPAx-I variant, i.e. an antisymmetrized kernel, however multiplied only with direct bielectronic integrals, without antisymmetrization. From the 4 different flavours directly accessible through the combinations of antisymmetrization (dRPA, RPAx, $-\mathrm{I},-\mathrm{II})^{34}$ this variant has been shown to be the most reliable for several test sets. ${ }^{35}$

All of the aforementioned correlation methods have in common that the correlation energy is expressed as a sum over amplitudes of doubly-excited determinants and bielectronic integrals (note that this is not the case for MP4 or $\operatorname{CCSD}(\mathrm{T})$ for instance). This allows (for two fragments) for a decomposition in 6 different classes of the excitations if orbitals are localized on different fragments. ${ }^{36-39}$ Two separate mono-excitations form a contribution that we may qualify as "dispersion". It has been shown ${ }^{40}$ that in a context which does not include strong electrostatic fields or important charge-transfer situations, the overall long-range correlation contribution to the correlation energy is already well described by taking into account only this part of the whole sum. A separate calculation of the monomer correlation energy is not necessary. A further step, which we did not apply here, is given by solving the RPA equations in the same-monomer monoexcitations only, thus reducing again considerably the necessary computational resources. ${ }^{40}$

\section{The method of increments}

The method of increments is a scheme for the calculation of energy-related properties for extended systems. ${ }^{41,42}$ In its traditional formulation by Stoll, ${ }^{2-4}$ the electron correlation energy of a system is expressed exactly in a many body expansion

$$
E_{\mathrm{corr}}=\sum_{I} \epsilon_{I}+\sum_{I} \sum_{J>I} \Delta \epsilon_{I J}+\sum_{I} \sum_{J>I} \sum_{K>J>I} \Delta \epsilon_{I J K}+\cdots
$$


The one-center increments, $\epsilon_{I}$, are defined as the correlation energy of the electrons in one group of occupied orbitals denoted by $I$. In practice these groups are defined by geometrical criteria, e.g. including all orbitals from one well defined fragment of the total system, called centers, e.g. one molecule, atom or bond. The two-center increments, $\Delta \epsilon_{i j}$, represent non-additive parts of the correlation energy from correlating all electrons from two fragments at the same time:

$$
\Delta \epsilon_{I J}=\epsilon_{I J}-\epsilon_{I}-\epsilon_{J}
$$

with $\epsilon_{I J}$ being the total correlation energy of fragments $I$ and $J$, which also includes contributions from the individual groups which have to be subtracted to avoid double counting of these terms. Higher order increments, such as three-center increments,

$$
\Delta \epsilon_{I J K}=\epsilon_{I J K}-\Delta \epsilon_{I J}-\Delta \epsilon_{I K}-\Delta \epsilon_{J K}-\epsilon_{I}-\epsilon_{J}-\epsilon_{K}
$$

are defined likewise by subtracting from the highest-order correlation energies all lower-order increments involving the corresponding indices. In principle only the full many-body expansion yields the correct electron correlation energy, however, when using localized orbitals even truncated expansions including all one, two and a few three-center increments up to a certain cut-off distance between fragments $I, J, K$, often recover more than $98 \%$ of the full electron correlation energy. ${ }^{42,43}$

Energy differences can easily be expressed in a similar way and often converge even faster with respect to the order of increments and the cut-off distance. ${ }^{43-48}$ In an expansion of an adsorption energy :

$$
E_{\mathrm{ads}}=\sum_{I} \eta_{I}+\sum_{I} \sum_{J>I} \eta_{I J}+\sum_{I} \sum_{J>I} \sum_{K>J} \eta_{I J K}+\cdots
$$

the adsorption energy increments, $\eta_{I}, \eta_{I J}$, and so on, represent firstly the change in correlation energy increments $\epsilon_{I}, \Delta \epsilon_{I J}, \ldots$ when adsorbing e.g. an atom, $\mathrm{A}$, at a surface:

$$
\eta_{I}=\epsilon_{I \in \text { surface }}^{\mathrm{A}+\text { surface }}-\epsilon_{I \in \text { surface }}^{\text {surface }}
$$

and secondly the dispersion interaction between the atom and the surface:

$$
\eta_{I J}=\Delta \epsilon_{I \in \mathrm{A}, J \in \text { surface }}^{\mathrm{A}+\text { surface }}
$$

For weakly bound systems - such as investigated in this study - the latter type of adsorption energy increment represents the major part of the atom-surface interaction, while the influence of the adsorbent on electrons in the surface - covered mainly by all $\eta_{I}$ and $\eta_{I J}$ of the first type 
- is of minor importance. For such a system we propose and test here as well a dispersion-only approximation which combines two ideas: first, we neglect all purely intra-fragment increments

$$
E_{\text {ads }} \approx \sum_{I \in \mathrm{A}} \sum_{J \in \text { surface }} \eta_{I J}+\sum_{I \in \mathrm{A}} \sum_{J, K \in \text { surface, }, J<K} \eta_{I J K}+\sum_{I, J \in \mathrm{A}, I<J} \sum_{K \in \text { surface }} \eta_{I J K}
$$

and secondly, the same simplification is applied to the calculation of the inter-fragment increments, where we correct the three-center increments only for the two-center inter-fragment correlation energies:

$$
\begin{aligned}
\eta_{I J} & =\epsilon_{I J} & & I \in \mathrm{A}, J \in \text { surface } \\
\eta_{I J K} & =\epsilon_{I J K}-\epsilon_{I J}-\epsilon_{I K} & & I \in \mathrm{A}, J, K \in \text { surface } \\
\eta_{I J K} & =\epsilon_{I J K}-\epsilon_{I K}-\epsilon_{J K} & & I, J \in \mathrm{A}, K \in \text { surface }
\end{aligned}
$$

The latter approximation - which we only employed in combination with RPA - avoids thus the calculation of any single-fragment and intra-fragment terms and all contributions are obtained from calculations on the complete system only.

Due to the rapid $1 / r^{6}$ decay of the electron dispersion interaction, the incremental expansion of adsorption energies converges even quicker than the electron correlation energy expansion with respect to the distances between the atom and the surface, and between surface atoms and the adsorption site. Axilrod-Teller dispersion - i.e. 3-body dispersion - decaying with $1 / r^{9}$, is often well recovered by a very few 3 -center increments between the atom and the surface.

\section{COMPUTATIONAL PROCEDURE}

For incremental MP2 or $\operatorname{CCSD}(\mathrm{T})$ calculations the occupied canonical Hartree-Fock orbitals are localized with the Foster-Boys ${ }^{49}$ procedure before ordering them in groups. Before performing the post Hartree-Fock calculation for one increment, the part of the Fock matrix that corresponds to the orbitals in which electrons are correlated for this increment is diagonalized again ("partial recanonicalization"), allowing for the use of standard implementations of post Hartree-Fock methods with canonical orbitals. All increments are constructed from the same set of localized occupied and canonical virtual orbitals of the whole system.

For the RSHDFT calculations we localize the orbitals in a different way, in several steps, allowing for a unique attribution of each orbital to each fragment: monomers are first calculated in the respective monomer and the dimer basis, and the occupied valence orbitals of the second calculation are localized with the Foster-Boys procedure. From the first set the (canonical) virtual orbitals are taken, and from the second set the (localized) occupied orbitals, serving as starting orbitals 
for an orbital optimization through a Singles-CI procedure of the complete dimer system. We showed elsewhere ${ }^{26}$ that the (approximate) Singles-CI procedure is usable in a DFT framework as an alternative to a standard iterative Kohn-Sham procedure, avoiding thus delocalizations due to the diagonalization of the Fock or Kohn-Sham matrix. The obtained dimer orbitals have the best overlap with the starting orbitals, and, in this way we can identify and compare individually orbital contributions to the total correlation energy.

After a full four-index transformation the excitation space is selected according to the intended respective increments, and an RPA correlation energy is calculated, adding to the RSH-DFT interaction energy the missing long-range correlation energy.

The core electrons of the metal atoms were described by scalar relativistic pseudopotentials, another field of interest of Andreas Savin..$^{50}$ Medium-size pseudopotentials ${ }^{51}$ of the Stuttgart group were employed throughout, leaving 20 electrons on the metal atom ( $n-1$ spd and $n s$ electrons). For the RSHDFT calculations also the $1 s$ electrons of the carbon atoms are treated through a pseudopotential. ${ }^{52}$ Unless otherwise stated, the corresponding polarized valence-double-zeta basis sets ( $5 s 5 p 4 d 2 f$ for the metal atoms, $2 s 2 p 1 d$ for carbon) are used throughout. ${ }^{51-53}$ The explicitly treated metal electrons form one single center of which only the 12 valence electrons $\left(d^{10} s^{2}\right)$ are correlated.

For some of the calculations with the $\mathrm{C}_{24}$ fragment all-electron basis sets were used for the carbon atoms. As an all-electron aug-cc-pvdz basis set ${ }^{54}$ revealed to be too demanding, the inner circle of the $\mathrm{C}_{24}$ fragment was described with the aug-cc-pvdz basis set $(4 s 3 p 2 d)$ and the augmentation functions were dropped for the outer circle $(3 s 2 p 1 d)$, leading to a total number of 504 contractions. Hydrogen is described by a $2 s 1 p$ basis set. ${ }^{54}$

\section{RESULTS}

\section{A. Full systems}

For reference, we first present counterpoise-corrected canonical-orbital calculations for the metal atoms adsorbing at the hollow-site of a $\mathrm{C}_{6}$ hydrogen-terminated, ideally flat graphene fragment the simplest model for our system. The experimental structure of graphene has a lattice constant of $2.4612 \AA$, and a unique $\mathrm{C}-\mathrm{C}$ distance of $1.42097 \AA$ results, which is a little longer than in a benzene molecule $(139 \mathrm{pm})$, reflecting the $\mathrm{C}-\mathrm{C}$ bond order, lower in graphene than in benzene. The $\mathrm{C}-\mathrm{H}$ bond length is set to $1.09621 \AA$. The metal atom is placed above the center of the ring, 
forming thus a set-up with $\mathrm{C}_{6 v}$ symmetry.

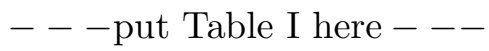

We see from Table I that the equilibrium distance follows the order $\mathrm{Cd}>\mathrm{Hg}>\mathrm{Zn}$. This ordering is well-known already from the corresponding metal dimers. ${ }^{56}$ We recognize that MP2 overbinds and $\operatorname{CCSD}(\mathrm{T})$ gives slightly lower binding energies than the range-separated DFT approach. The contribution of the correlation energy is smaller (compared to the total interaction energy) for the RSHDFT approach than for MP2 or $\operatorname{CCSD}(\mathrm{T})$, as already some of the correlation contributions are accounted for in the density-functional part. We see from the data as well that the dispersion approximation yields about the same results as the complete RSH+RPA calculations, showing the validity of this approach in the present case.

Applying a larger triple-zeta basis set for Zn reveals that indeed the range-separated DFT + RPA comes closer to the basis-set limit than RHF + MP2. As shown by Franck et al. ${ }^{57}$ RSHDFT results should be extrapolated toward the complete basis set limit via an exponential expression, needing however at least three points. Since we calculated only double and triple-zeta results, we use the two-parameter formula $E(X)=E_{\infty}+B / X^{3}$ of Helgaker et al. ${ }^{55}$ for basis set extrapolations for both, HF + MP2 and RSHDFT + RPA cases.

\section{B. Orbital-generating RHF and RSHLDA calculations}

As calculations are carried out for hydrogen-terminated finite fragments of graphene, we require an extrapolation scheme for estimating the interaction energy with an infinite graphene sheet. For studying the adsorption on three different sites ("on top", "hollow", "bridge") different models of a graphene sheet may be used. Either all calculations are done on the same finite fragment, breaking thus the symmetry of the infinite system, or different fragments of graphene are used for each adsorption site, maintaining the highest possible symmetry. However, when associating three $\mathrm{C}_{6}$ rings with a three-fold symmetry for the on-top adsorption, the number of $\pi$ electrons in the fragment becomes odd, leading to a doublet ground state. We therefore prefer to study all three adsorption sites in the same fragment with a central $\mathrm{C}_{6}$ unit. 
The next larger, reasonable fragments are a $\mathrm{C}_{24}$ (coronene-like) and a $\mathrm{C}_{54}$ fragment. For all three we calculate the adsorption of $\mathrm{Zn}$ at different distances, and we observe that the difference between $\mathrm{C}_{24}$ and $\mathrm{C}_{54}$ becomes negligible, see Figure 1, which leads us to the conclusion that the RHF interaction energy is already converged for the $\mathrm{C}_{24}$ fragment. Hence no further extrapolation is used for estimating the interaction energy at the RHF and RSH-DFT level with the infinite graphene sheet.

In the same figure we show the interaction curves at the RSH-DFT level for the $\mathrm{C}_{24}$ fragment for all three metals for the hollow site, which present again an ordering $\mathrm{Zn}-\mathrm{Hg}-\mathrm{Cd}$. For $\mathrm{Zn}$ we explore as well the two other adsorption sites (on top and bridge), with the result that all three positions appear to be equivalent at this level of calculation.

\section{Correlation contributions via increments}

In section III A we calculated the correlation energy for the full system as the difference between the fragments and the interacting system $(\mathrm{RHF}+\mathrm{CCSD}(\mathrm{T}))$ or directly via the dispersion contribution only (RSHLDA + RPAx-I). This was possible for the $\mathrm{C}_{6}$ fragment as a medium-sized system. For the $\mathrm{C}_{24}$ model of graphene this direct approach becomes too costly due to the steep increase in computational demand with system size. Instead we resort to incremental calculations of the correlation energy.

As stated above, the orbitals are localized with the Foster-Boys procedure, either a posteriori (RHF) or on each fragment separately before determining the orbitals of the full system through the Single-CI procedure (RSHLDA). This yields for the $\mathrm{C}_{24}$ fragment the pattern of double and single bonds as depicted in the upper left panel of Figure 2.

For calculating the RHF or RSHLDA interaction energy the localization pattern is irrelevant, however a choice has to be made for the incremental calculations, as the number of electrons to be correlated depends on the orbital groups chosen (two for a single bond, four for a double bond). In the following we use the obtained Foster-Boys pattern which may be extended to larger finite fragments of graphene, and even to the infinite sheet as indicated in the upper right panel of figure 2 .

$$
--- \text { put Figure } 2 \text { here }---
$$

Having decided on one "Lewis" pattern, we can count the number of individual increments 
which have to be calculated for the hollow adsorption site, exploiting the symmetry of the model. We find in total 5 one-center, 50 two-center and 407 unique three-center increments, the latter for instance representing the ensemble of all 4495 three-center increments if symmetry were ignored. From these symmetry-unique increments only 4 two-center and 46 three-center increments are actually to be considered for the dispersion-only approximation, the others being intra-fragment increments or presenting excitations from one to the other fragment.

Since without the dispersion-only approximation the number of increments is still large, we analyze the decay of the increments with respect to the metal-graphene distance in the $\mathrm{Zn} / \mathrm{C}_{6}$ system to identify increments that could be neglected as contributing only little to the overall result. From the data in Table II, left side, we conclude that three-center intra-fragment increments may be dropped. Thus we do not calculate them for the $\mathrm{C}_{24}$ system and consider only 28 out of the remaining 46 three-center inter-fragment increments which include at least one double bond, correlating 18 or 20 electrons for each of these (the metal and one or two double bonds). In the case of RSHLDA + RPA we apply the dispersion-only approximation: (1) calculating only the 2-center and the previously selected 283 -center terms and (2) taking from the correlation energy only those contributions which can be decomposed into two monoexcitations on either fragment, needing no corrections from one-center increments to exclude intra-fragment contributions to the correlation energies. This leaves us in this case with only 4 two-center and 28 three-center increments that are actually to be considered.

$$
--- \text { put Table II here }---
$$

The overall interaction energies and equilibrium metal-graphene distances are shown in the upper part of Table IV (vide infra). We observe that the ordering of the energies remains the same as for the $\mathrm{C}_{6}$ fragment, however $\mathrm{Cd}$ and $\mathrm{Hg}$ result are in very similar positions of the minimum. Figure 3 presents for the $\mathrm{Zn} / \mathrm{C}_{24}$ system the contributions of each class of increments at a metalgraphene distance of $3.5 \AA$, illustrating the importance of the three-center terms, which we may estimate for the RPAx-I case to be about $20 \%$ of the two-center increments, but with opposite sign. 


\section{Extrapolation to infinite systems}

From the obtained results for the $\mathrm{C}_{24}$ model we may extrapolate towards the infinite graphene sheet. In section IIIB we showed that the Hartree-Fock calculations are converged for $\mathrm{C}_{24}-$ exchange interactions decay exponentially with the distance and induction should be absent as the metal atoms do not bear permanent moments - however, correlation, in particular dispersion, decays much slower, as $r^{-6}$.

By now we calculated explicitly the contribution of each individual metal-C-C-bond interaction as one, two- and three-center increments for the metal- $\mathrm{C}_{24}$ system. In the following, two complementary ways are explored for extrapolating these results to an adsorption energy of a metal atom on an infinite graphene sheet: in the first place directly from the data already obtained, and secondly by accumulating simplified calculations.

\section{Extrapolation of the Increments}

In the $\mathrm{C}_{24}$ fragment we have radial double bonds in the inner ring, and circular double bonds in the outer ring, leading to two distinct curves to be fitted. The single bonds are all mainly oriented circularly.

If one traces the contribution of each type of bond in the $\mathrm{C}_{24}$ fragment against the distance of its bond center to the metal, one may first extrapolate these to the next two larger fragments $\mathrm{C}_{54}$ and $\mathrm{C}_{96}$ of a graphene sheet, and further to an infinite system, by the help of a double-logarithmic plot assuming asymptotically a simple power law $y(r)=C r^{m}$. Indeed, the double-logarithmic plot shows straight lines (see Figure 4 for one example), and the fit yields parameters with small errors, as assembled in Table III.

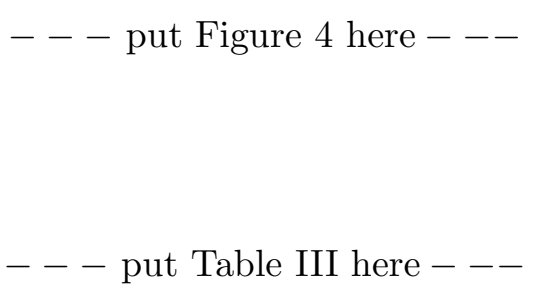

In Table III we distinguish "inner" and "outer" bonds, as present in the $\mathrm{C}_{24}$ fragment, corresponding to the two distinct decays in the log-log plot. For the extrapolation we use the increments obtained from the $\mathrm{C}_{24}$ calculations, and we continue the pattern of single and double bonds of the 
infinite graphene sheet to $25 \AA$, by using the average of the exponents and pre-factors as extrapolation function

$$
f(r)=\frac{1}{2}\left(C_{1}+C_{2}\right) r^{\frac{1}{2}\left(m_{1}+m_{2}\right)} \quad
$$

We accumulate the extrapolated increments to a convergent correlation contribution to the binding energy. To this we add the RHF or RSH contribution of the binding on the $\mathrm{C}_{24}$ fragment, which we considered converged with the size of the fragment (see section IIIB).

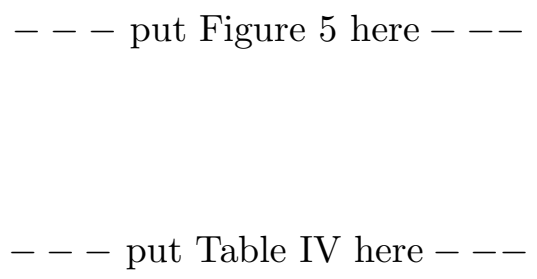

In Figure 5 we compare the three metals for the hollow adsorption site, once for the $\mathrm{C}_{24}$ fragment (hollow symbols) treated in section IIIC, and once for the extrapolated data (full symbols). For $\mathrm{Zn}, \mathrm{CCSD}(\mathrm{T})$ and RPA + RSHDFT yield very similar results, and Cd and Hg lie very close. The order of the latter two appears to be inverse with respect to the $\mathrm{C}_{24}$ fragment. Additionally, we see in Table IV that range-separated hybrid DFT together with the RPAx-I correlation part and the dispersion approximation, leads to similar extrapolated data as the $\operatorname{CCSD}(\mathrm{T})$ approach. However, available computer resources did not allow for any further extension of this study to the other two metals and other adsorption sites at the RSHLDA + RPAx-I level, but we are confident that results will be similar to those of Zn.

\section{Simplification}

With a simpler model — inspired by the original work from Stoll ${ }^{2-4}$ — we may overcome the resource restrictions mentioned above. We showed in Table II that we may indeed use the dispersion part of the two-center and three-center increments in the RSHLDA-RPA context. If we neglect for the moment all three-center increments, we only need a metal atom and one single ethane $\left(\mathrm{C}_{2} \mathrm{H}_{6}\right)$ or ethene $\left(\mathrm{C}_{2} \mathrm{H}_{4}\right)$ molecule, cut as the previous fragments from the graphene sheet and saturated with hydrogens, for modeling a single or double bond, respectively. Then we only have to add up all the different contributions of symmetry-equivalent bonds. The difficulties due to different spin functions of Lewis structures of graphene fragments do not arise any more. We may work directly 
with a supposedly infinite graphene structure with an underlying Lewis structure with equivalent six-rings, see Figure 2, lower part.

Calculating the individual contributions of each bond in the graphene sheet one by one yields indeed asymptotically a $1 / R^{6}$ decay of the contributions, as shown in Figure 6 , and the cumulative sum converges smoothly to its asymptotic value, see Figure 7.

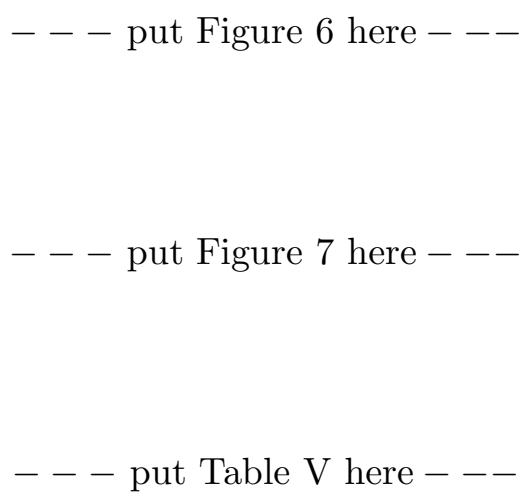

In Table V, two leftmost columns, we show the binding energies obtained by accumulating the dispersion part of the correlation energies of the individually calculated two-body increments. As expected, this binding energy is far too high with respect to the values obtained in the previous section. A very crude approximation to the final binding will be to scale the correlation part by the previously obtained $20 \%$, which displaces slightly the equilibrium distance, and binding energies closer to the values already reported. For having a more reliable approximation than a global scaling, we select all 3-body increments which are composed of three adjacent carbon atoms (distorted $\mathrm{C}_{3} \mathrm{H}_{8}$ or $\mathrm{C}_{3} \mathrm{H}_{6}$ molecules with two or one single bond), and calculate their contribution, again relying on the dispersion approximation. Of these we correlate only the two or three $\mathrm{C}-\mathrm{C}$ bonds, localized with the Foster-Boys procedure, and ignoring for the correlation calculation the $\mathrm{C}-\mathrm{H}$ bonds. The obtained corrections are larger than the estimated $20 \%$, which results in overall binding energies weaker than for the previous extrapolation scheme.

\section{CONCLUSION}

In the present study of the adsorption of $\mathrm{Zn}, \mathrm{Cd}$ or $\mathrm{Hg}$ on graphene we see that range-separated density-functional theory, complemented by long-range correlation contributions, is a reliable tool for studying adsorption problems governed by dispersion interactions. For rendering the method applicable to this kind of extended system, the correlation part was split into small increments 
calculated separately with localized orbitals, and results on small model systems $\left(\mathrm{C}_{6}, \mathrm{C}_{24}\right)$ could be extrapolated successfully towards an infinite graphene sheet. The first step, the mean-field calculation, converges already for the hydrogen-saturated $\mathrm{C}_{24}$ fragment of the graphene sheet, as interactions decay exponentially with the interatomic distance.

The proposed reduction of the correlation part to independent calculations on even smaller subsystems, relying only on 2-center increments and the dispersion approximation is too crude an approximation in the present case. The ensemble of repulsive three-center increments has to be included in the calculations, which can be estimated to reduce by about $20 \%$ the correlation part of the interaction energy. Considering connected 3-center increments explicitly a slightly larger correction is obtained..

The two extrapolation approaches to the correlation energy of an infinite graphene sheet yield similar results, with different advantages and disadvantages. The first, the extrapolation from a $\mathrm{C}_{24}$ fragment, relies on a perfectly controllable system of reasonable size, needs however one single, demanding calculation for obtaining common orbitals, and subsequently, large files to handle before selection and truncation for the incremental correlation calculations. The latter approach, subdivided in individual and independent, thus perfectly parallelizable, calculations, needs much less resources in an astonishing simple approach, but the accuracy with respect to the chosen system - a conducting graphene sheet - is reduced compared to the first approach.

All three metals are bound with similar binding strength of about $150(\mathrm{Zn})-200 \mathrm{meV}(\mathrm{Hg})$, in good comparison to literature values, ${ }^{8,13}$.

\section{ACKNOWLEDGEMENTS}

The calculations have been carried out in the laboratory of theoretical chemistry in Paris and ZeDat in Berlin. Additional computing resources, furnished by the computing platform jarvis@IP2CT of the research federation IP2CT in Paris, are gratefully acknowledged. P.R. expresses his gratitude and warmest regards for numerous outstanding discussions with A. Savin in Paris and elsewhere. For the present project the advice and practical help of J. Toulouse and B. Mussard in Paris were of great value, as well as discussions with H. Stoll in Stuttgart about recently published work. ${ }^{11}$ The stay of F.W. in Paris was possible through a grant of the FU Berlin via the PROMOS program. 
C.M. acknowledges support by the DFG GRK 1582 "Fluorine as a key element".

1 A. Savin, "On degeneracy, near-degeneracy and density functional theory", in : J. Seminario, ed., "Recent development and applications of Density Functional Theory", Elsevier, Amsterdam, (1996) 327

2 H. Stoll, "Correlation energy of diamond", Phys.Rev.B, 46 (1992) 6700

3 H. Stoll, "The correlation energy of crystalline silicon", Chem.Phys.Lett., 191 (1992) 548

4 H. Stoll, "On the Correlation Energy of Graphite", J.Chem.Phys., 97 (1992) 8449

5 K.T. Chan, J.B. Neaton, M.L. Cohen, "First-principles study of metal adatom adsorption on graphene", Phys.Rev.B, 77 (2008) 235430

6 Y. Mao, J. Yuan, J. Zhong, "Density functional calculation of transition metal adatom adsorption on graphene", J.Phys.Cond.Mat., 20 (2008) 115209

7 P.V.C. Medeiros, F. de Brito Mota, A.J.S. Mascarenhas, C.M.C. de Castilho, "Adsorption of monovalent metal atoms on graphene: a theoretical approach", Nanotech., 21 (2010) 115701

8 K. Nakada, A. Ishii, "Migration of adatom adsorption on graphene using DFT calculation", Sol.Stat.Comm., 151 (2011) 13

9 T. Zhang, L. Zhu, S. Yuan, J. Wang, "Structural and Magnetic Properties of 3d Transition-Metal-Atom Adsorption on Perfect and Defective Graphene: A Density Functional Theory Study", Chem.Phys.Chem., 14 (2013) 3483

10 S.Y. Davydov, "Transition metal and rare-earth metal atoms on single-layer graphene: Estimations of the charge transfer and adsorption energy", Phys.Sol.Stat., 55 (2013) 1536

11 M.P. de Lara-Castells, A.O. Mitrushchenkov, H. Stoll, "Combining density functional and incremental post-Hartree-Fock approaches for van der Waals dominated adsorbate-surface interactions: $\mathrm{Ag}_{2}$ /graphene", J.Chem.Phys., 143 (2015) 102804

12 V.N. Kotov, B. Uchoa, V.M. Pereira, F. Guinea, A.H. Castro Neto, "Electron-Electron Interactions in Graphene: Current Status and Perspectives", Rev.Mod.Phys., 84 (2012) 1067

13 M. Manadé, F. Vines, F. Illas, "Transition metal adatoms on graphene: A systematic density functional study", Carbon, 95 (2015) 525

14 B.G. Johnson, P.M.W. Gill, J.A. Pople, "The performance of a family of density functional methods", J.Chem.Phys., 98 (1993) 5612

15 J.A. Pople, P.M.W. Gill, B.G. Johnson, "KohnSham density-functional theory within a finite basis set", J.Chem.Phys., 199 (1992) 557

16 S. Grimme, "Semiempirical GGA-type density functional constructed with a long-range dispersion correction", J.Comp.Chem., 27 (2006) 1787

17 S. Grimme, J. Antony, S. Ehrlich, H. Krieg, "A consistent and accurate ab initio parameterization of density functional dispersion correction (DFT-D) for the 94 elements H-Pu", J.Chem.Phys., 132 (2010) 
154104

18 S. Grimme, S. Ehrlich, L. Goerigk, "Effect of the damping function in dispersion corrected density functional theory", J.Comp.Chem., 32 (2011) 1456

19 W. Kohn, Y. Meir, D.E. Makarov, "van der Waals energies in density functional theory", Phys.Rev.Lett., $80(1998) 4153$

20 H. Iikura, T. Tsuneda, T. Yanai and K. Hirao, "A long-range correction scheme for generalized-gradientapproximation exchange functionals", J.Chem.Phys., 115 (2001) 3540

21 O.A. Vydrov, G.E. Scuseria, "Assessment of a long-range corrected hybrid functional", J.Chem.Phys., 125 (2006) 234109

22 Th.M. Henderson, A.F. Izmaylov, G.E. Scuseria, A. Savin, "Assessment of a Middle-Range Hybrid Functional", J.Chem.Theor.Comp., 4 (2008) 1254

23 J. Heyd, G.E. Scuseria, M. Ernzerhof, "Hybrid functionals based on a screened Coulomb potential", J.Chem.Phys., 118 (2003) 8207

24 T. Yanai, D.P. Tew, N.C. Handy, "A new hybrid exchangecorrelation functional using the Coulombattenuating method (CAM-B3LYP", Chem.Phys.Lett., 393 (2004) 51

25 J. Toulouse, A. Savin, H.-J. Flad, "Short-range exchange-correlation energy of a uniform electron gas with modified electron-electron interaction", Int.J.Quant.Chem., 100 (2004) 1047

26 P. Reinhardt, J.P. Piquemal, A. Savin, "Singles-CI localized DFT orbitals for analyzing intermolecular interactions", J.Theor.Chem.Comp., 4 (2008) 2020

27 J.G. Ángyán, I.C. Gerber, A. Savin, J. Toulouse, "van der Waals forces in density functional theory: Perturbational long-range electron interaction corrections", Phys.Rev.A, 72 (2005) 012510

28 E. Goll, H.-J. Werner, H. Stoll, "A Short-Range Gradient-Corrected Density Functional in Long-Range Coupled-Cluster Calculations for Rare-Gas Dimer", Phys.Chem.Chem.Phys., 7 (2005) 3917

29 E.A. Hylleraas, "Über den Grundterm der Zweielektronenprobleme von $\mathrm{H}^{-}, \mathrm{He}, \mathrm{Li}^{+}, \mathrm{Be}^{++}$usw.", Z.Phys., 65 (1930) 209

30 D. Pines, D. Bohm, "A Collective Description of Electron Interactions: II. Collective versus Individual Particle Aspects of the Interactions", Phys.Rev., 85 (1952) 338

31 T.D. Bouman, B. Voigt, A.E. Hansen, "Optical activity of saturated ketones. Ab initio localized orbital analysis of a model ketone in the random-phase approximation", J.Am.Chem.Soc., 101 (1979) 550

32 J. Toulouse, W. Zhu, J.G. Ángyán, A. Savin, "Range-separated density-functional theory with the random phase approximation: Detailed formalism and illustrative applications", Phys.Rev.A, 82 (2010) 032502

33 J. Toulouse, W. Zhu, A. Savin, G. Jansen, J.G. Ángyán, "Closed-shell ring coupled cluster doubles theory with range separation applied on weak intermolecular interactions", J.Chem.Phys., 135 (2011) 084119

34 J.G. Ángyán, R.-F. Liu, J. Toulouse, G. Jansen, "Correlation energy expressions from the adiabaticconnection fluctuation-dissipation theorem approach", J.Chem.Theor.Comp., 7 (2011) 3116 
35 W. Zhu, J. Toulouse, A. Savin, J. Ángyán, "Range-separted density-functional theory with random phase approximation applied to non-covalent intermolecular interactions", J.Chem.Phys., 132 (2010) 244108

36 In a general set-up we may have double excitations implication at maximum 4 different fragments.

37 M. Schütz, G. Rauhut, H.-J. Werner, "Local Treatment of Electron Correlation in Molecular Clusters: Structures and Stabilities of $\left(\mathrm{H}_{2} \mathrm{O}\right)_{n}, n=2-4 . "$, J.Phys.Chem.A, 102 (1998) 5997

38 N. Runeberg, M. Schütz, H.-J. Werner, "The aurophilic attraction as interpreted by local correlation methods", J.Chem.Phys., 110 (1999) 7210

39 P. Reinhardt, "The decomposition of intermolecular interaction energies in localized orbitals - critical analysis and an invariance", Chem.Phys.Lett., 370 (2003) 338

40 E Chermak, B Mussard, J Ángyán, P Reinhardt, "Short range DFT combined with long-range local RPA within a range-separated hybrid DFT framework", Chem.Phys.Lett., 550 (2012) 561

41 B. Paulus, "The method of increments - a wavefunction-based ab initio correlation method for solids", Phys.Rep., 428 (2006) 1

42 C. Müller, B. Paulus, "Wavefunction-based electron correlation methods for solids", Phys.Chem.Chem.Phys., 14 (2012) 7605

43 B. Paulus, K. Rościszewski, "Application of the method of increments to the adsorption of $\mathrm{H}_{2} \mathrm{~S}$ on graphene", Int.J.Quant.Chem., 109 (2009) 3055

44 E. Voloshina, D. Usvyat, M. Schütz, Y. Dedkov, B. Paulus, "On the physisorption of water on graphene: a CCSD(T) study", Phys.Chem.Chem.Phys., 13 (2011) 12041

45 C. Müller, B. Herschend, K. Hermansson, B. Paulus, "Application of the method of increments to the adsorption of $\mathrm{CO}$ on the $\mathrm{CeO}_{2}(110)$ surface", J.Chem.Phys., 128 (2008) 214701

46 C. Müller, B. Paulus, K. Hermansson, "Ab initio calculations of CO physisorption on ceria (111)", Surf.Sci., 603 (2009) 2619

47 C. Müller, K. Hermansson, B. Paulus, "Electron correlation contribution to the $\mathrm{N}_{2} \mathrm{O} /$ ceria (111) interaction", Chem.Phys., 362 (2009) 91

48 L. Hammerschmidt, L. Maschio, C. Müller, B. Paulus, "Electron Correlation at the $\mathrm{MgF}_{2}(110)$ Surface: A Comparison of Incremental and Local Correlation Methods", J.Chem.Theor.Comp., 11 (2015) 252

49 J.M. Foster, S.F. Boys, "Canonical Configurational Interaction Procedure", Rev.Mod.Phys., 32 (1960) 300

50 A. Savin, U. Wedig, H. Stoll, H. Preuss, "The correlated electron density of the Li atom - a test for density functionals and semiempirical pseudopotentials", Chem.Phys.Lett., 92 (1982) 503

51 D. Figgen, G. Rauhut, M. Dolg, H. Stoll, "Energy-consistent pseudopotentials for group 11 and 12 atoms: adjustment to multi-configuration Dirac-Hartree-Fock data", Chem.Phys., 311 (2005) 227

52 A. Bergner, M. Dolg, W. Kuechle, H. Stoll, H. Preuss, "Ab-initio Energy-Adjusted Pseudopotentials for Elements of Groups 13-17", Mol.Phys., 80 (1993) 1431

53 K.A. Peterson, C. Puzzarini, "Systematically convergent basis sets for transition metals. II. Pseudopotential-based correlation consistent basis sets for the group $11(\mathrm{Cu}, \mathrm{Ag}, \mathrm{Au})$ and $12(\mathrm{Zn}, \mathrm{Cd}$, 
Hg) elements", Theor.Chem. Acc., 114 (2005) 283

54 T.H. Dunning, Jr., "Gaussian Basis Sets for Use in Correlated Molecular Calculations. I. The Atoms Boron through Neon and Hydrogen", J.Chem.Phys., 90 (1989) 1007

55 T. Helgaker, P.J. Jørgensen, J. Olsen, "Molecular Electronic Structure Theory", John Wiley and Sons, Chichester, UK, (2000)

56 L. Bucinsky, S. Biskupic, M. Ilcin, V. Lukes, V. Laurinc, "On Relativistic Effects in Ground State Potential Curves of $\mathrm{Zn}_{2}, \mathrm{Cd}_{2}$, and $\mathrm{Hg}_{2}$ Dimers. A CCSD(T) Study", J.Comp.Chem., 30 (2009) 65

57 O. Franck, B. Mussard, E. Luppi, J. Toulouse, "Basis convergence of range-separated density-functional theory", J.Chem.Phys., 142 (2015) 074107 


\begin{tabular}{|c|c|c|c|c|c|c|c|c|c|}
\hline & \multicolumn{3}{|c|}{$\mathrm{Zn}$} & \multicolumn{3}{|c|}{$\mathrm{Cd}$} & \multicolumn{3}{|c|}{$\mathrm{Hg}$} \\
\hline & $\begin{array}{l}\text { dist } \\
(\AA)\end{array}$ & $\begin{array}{c}\Delta E \\
(\mathrm{meV})\end{array}$ & $\begin{array}{r}\Delta E_{\text {Corr }} \\
(\mathrm{meV})\end{array}$ & $\begin{array}{l}\text { dist } \\
(\AA)\end{array}$ & $\begin{array}{c}\Delta E \\
(\mathrm{meV})\end{array}$ & $\begin{array}{c}\Delta E_{\text {Corr }} \\
(\mathrm{meV})\end{array}$ & $\begin{array}{l}\text { dist } \\
(\AA)\end{array}$ & $\begin{array}{c}\Delta E \\
(\mathrm{meV})\end{array}$ & $\begin{array}{r}\Delta E_{\text {Corr }} \\
(\mathrm{meV})\end{array}$ \\
\hline $\mathrm{RHF}+\mathrm{MP} 2$ & 3.37 & -101 & -146 & 3.44 & -123 & -216 & 3.35 & -147 & -263 \\
\hline $\mathrm{RHF}+\operatorname{CCSD}(\mathrm{T})$ & 3.62 & -53 & -106 & 3.72 & -59 & -100 & 3.65 & -64 & -110 \\
\hline RSHLDA + RPAx-I & 3.37 & -77 & -107 & 3.48 & -88 & -121 & 3.44 & -97 & -131 \\
\hline disp.approx. RSHLDA + RPAx-I & 3.39 & -81 & -108 & 3.49 & -94 & -126 & 3.44 & -105 & -139 \\
\hline pvtz RHF + MP2 & 3.23 & -155 & -286 & & & & & & \\
\hline pvtz RSHLDA + RPAx-I & 3.37 & -89 & -131 & & & & & & \\
\hline CBS RHF + MP2 & 3.17 & -179 & & & & & & & \\
\hline CBS RSHLDA + RPAx-I & 3.37 & -93 & & & & & & & \\
\hline
\end{tabular}

TABLE I. Reference calculations, for the hollow adsorption site, on a $\mathrm{C}_{6}$ graphene fragment. We give the equilibrium distance and the binding energies, and the correlation contribution. As the Hartree-Fock or DFT interactions are repulsive, the correlation contribution is larger than the complete interaction energy. The $\mathrm{C}-\mathrm{H}$ bonds of the substrate are included in the calculation of the correlation energy. CBS are extrapolated values of the $X=2$ and $X=3$ (pvdz and pvtz) curves with help of $E(X)=E_{\infty}+B / X^{3}$. 


\begin{tabular}{lc|rrr|rrr} 
& & \multicolumn{3}{|c|}{$\mathrm{C}_{6}$} & \multicolumn{3}{|c}{$\mathrm{C}_{24}$} \\
\hline Method & Group & $\mathrm{n}=1$ & $\mathrm{n}=2$ & $\mathrm{n}=3$ & $\mathrm{n}=1$ & $\mathrm{n}=2$ & $\mathrm{n}=3$ \\
\hline MP2 & $\eta_{\text {Metal }}$ & 8.7 & & & -10.1 & & \\
& $\eta_{\text {intra }}$ & 27.6 & 28.0 & 0.9 & 28.9 & -9.5 & n.c. \\
& $\eta_{\text {inter }}$ & & -385.7 & -16.5 & & -509.7 & -24.0 \\
\hline CCSD(T) & $\eta_{\text {Metal }}$ & 19.3 & & & 0.1 & & \\
& $\eta_{\text {intra }}$ & 36.0 & 29.7 & -2.8 & 38.0 & -11.5 & n.c. \\
& $\eta_{\text {inter }}$ & -374.3 & 58.5 & & -475.0 & 111.9 \\
\hline RPAx-I & $\eta_{\text {inter }}$ & -138.2 & 14.8 & & -273.8 & 68.5 \\
\hline
\end{tabular}

TABLE II. Convergence behavior of specific groups of increments. $\mathrm{Zn}$ is placed at $3.0 \AA$ above the $\mathrm{C}_{6}$ (left columns) or $\mathrm{C}_{24}$ (right columns) graphene fragment. $\eta_{\text {intra }}$ and $\eta_{\text {inter }}$ denote the sums of all incremental intra-fragment and inter-fragment contributions. All data are in meV. 


\begin{tabular}{l|cc|cc|cc} 
Method & Center Metal & $\begin{array}{c}\text { Slope } \\
m\end{array}$ & $\begin{array}{c}\text { Offset } \\
\log \mathrm{C}\end{array}$ & $\begin{array}{c}\text { Slope } \\
m\end{array}$ & $\begin{array}{c}\text { Offset } \\
\log \mathrm{C}\end{array}$ \\
& & & \multicolumn{2}{|c}{ inner } & \multicolumn{2}{|c}{ outer } \\
\hline $\operatorname{CCSD}(\mathrm{T})$ & $\mathrm{SB}$ & $\mathrm{Zn}$ & $-5.93(4)$ & $4.22(2)$ & $-6.51(1)$ & $4.613(8)$ \\
& $\mathrm{DB}$ & & $-6.00(1)$ & $5.094(6)$ & $-6.98(1)$ & $5.765(6)$ \\
\hline $\mathrm{CCSD}(\mathrm{T})$ & $\mathrm{SB}$ & $\mathrm{Cd}$ & $-5.88(3)$ & $4.30(2)$ & $-6.527(3)$ & $4.736(2)$ \\
& $\mathrm{DB}$ & & $-5.96(2)$ & $5.16(2)$ & $-6.923(6)$ & $5.827(4)$ \\
\hline $\operatorname{CCSD}(\mathrm{T})$ & $\mathrm{SB}$ & $\mathrm{Hg}$ & $-6.24(3)$ & $4.48(2)$ & $-6.780(8)$ & $4.879(5)$ \\
& $\mathrm{DB}$ & & $-6.31(2)$ & $5.35(2)$ & $-7.30(1)$ & $6.05(1)$ \\
\hline $\mathrm{RPAx}-\mathrm{I}$ & $\mathrm{SB}$ & $\mathrm{Zn}$ & $-5.96(9)$ & $4.17(7)$ & $-6.16(3)$ & $4.37(2)$ \\
& $\mathrm{DB}$ & & $-6.00(9)$ & $4.97(7)$ & $-6.21(2)$ & $5.14(2)$ \\
\hline
\end{tabular}

TABLE III. Parameters $m$ and $C$ of a simple power law $y(r)=C r^{m}$ for the contribution of each type of bond for the inter-fragment increments of the $\mathrm{C}_{24}$ systems and the hollow-place adsorption site; SB stands for "Single Bond" and DB for "Double Bond". Values were obtained from a linear fit using the last 4 data points of each series. The number in parentheses indicate the precision of the last digit of each entry. 


\begin{tabular}{l|c|c|ccc} 
Substrate & Method & distance $(\AA)$ & $E_{\mathrm{Ads}}$ & $E_{\mathrm{RHF} / \mathrm{RSH}}$ & $E_{\text {Corr }}$ \\
\hline Coronene & & & & & \\
$+\mathrm{Zn}$ & $\operatorname{CCSD}(\mathrm{T})$ & 3.46 & -104 & 121 & -227 \\
& $\mathrm{RPAx}-\mathrm{I}$ & 3.40 & -102 & 52 & -154 \\
$+\mathrm{Cd}$ & $\operatorname{CCSD}(\mathrm{T})$ & 3.50 & -129 & 117 & -246 \\
$+\mathrm{Hg}$ & $\operatorname{CCSD}(\mathrm{T})$ & 3.51 & -135 & 95 & -230 \\
\hline Graphene & & & & & \\
$+\mathrm{Zn}$ & $\operatorname{CCSD}(\mathrm{T})$ & 3.38 & -145 & 114 & -259 \\
& $\mathrm{RPAx}-\mathrm{I}$ & 3.37 & -140 & 58 & -197 \\
$+\mathrm{Cd}$ & $\operatorname{CCSD}(\mathrm{T})$ & 3.45 & -180 & 131 & -311 \\
$+\mathrm{Hg}$ & $\operatorname{CCSD}(\mathrm{T})$ & 3.47 & -175 & 104 & -279
\end{tabular}

TABLE IV. Optimum positions, interaction energies and contributions of mean-field and correlation to the adsorption energy, in meV, for all three metals in the hollow position, once for the Coronene and once for the extrapolated graphene sheet. See text for explanations. 


\begin{tabular}{l|ll|ll|ll} 
Metal & $\mathrm{d}$ & $E_{\text {Ads }}$ & $\mathrm{d}$ & $E_{\text {Ads }}$ & $\mathrm{d}$ & $E_{\text {Ads }}$ \\
\hline & \multicolumn{2}{|c|}{ without scaling } & with scaling & \multicolumn{2}{|l}{ with 3-center terms } \\
\hline $\mathrm{Zn}$ & 3.27 & -192 & 3.36 & -140 & 3.44 & -119 \\
$\mathrm{Cd}$ & 3.31 & -240 & 3.45 & -176 & 3.49 & -151 \\
$\mathrm{Hg}$ & 3.26 & -259 & 3.37 & -192 & 3.37 & -173
\end{tabular}

TABLE V. Interaction energies for the hollow adsorption site, as calculated as the sum of the RSHLDA interaction energy and the extrapolated RPAx-I long-range correlation contributions using the dispersion approximation. In the left part we give the results for bare two-center fragments, in the middle we use scaled ones (by a factor of 0.80), and for the third double column we include the calculated repulsive 3-center contributions. All data are in $\AA$ and $\mathrm{meV}$. 


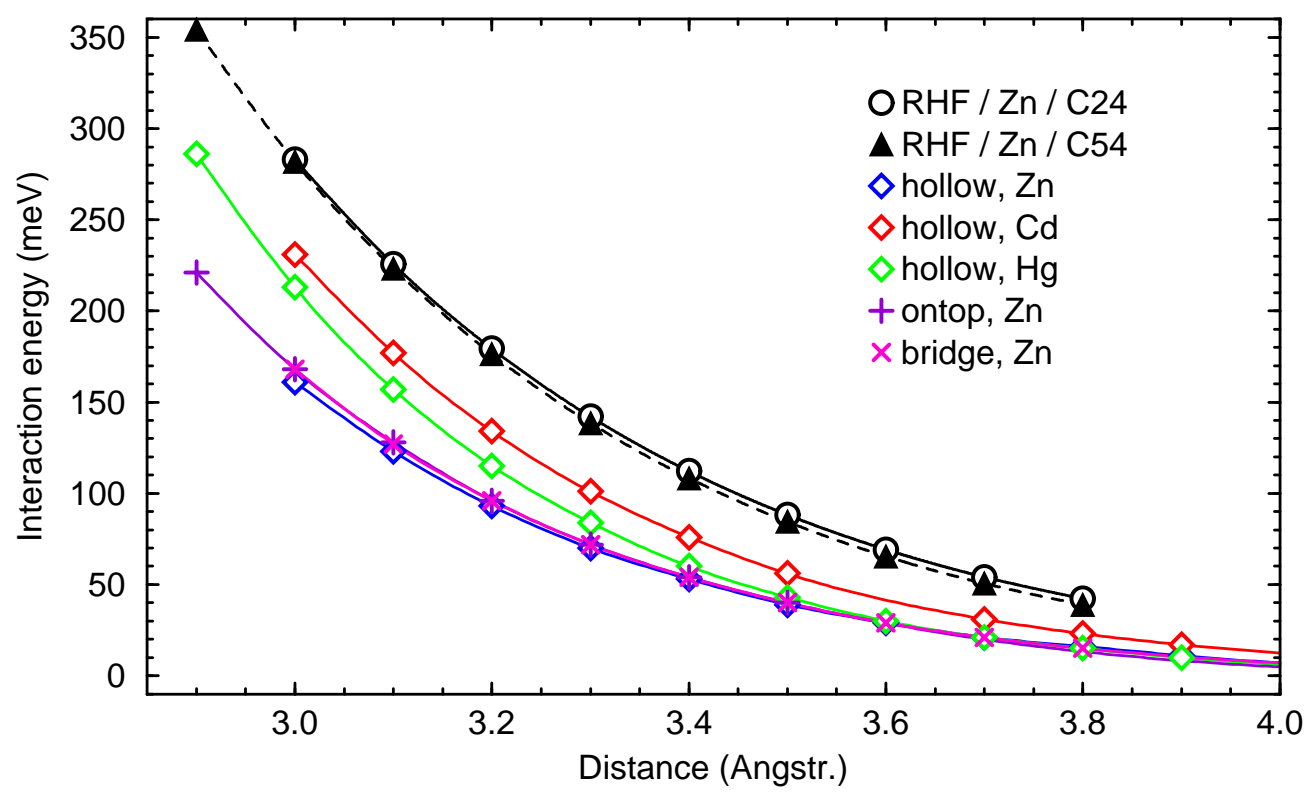

FIG. 1. Dependence of the RHF/RSHLDA repulsive interaction energies on the distance to the graphene fragment. First two lines (RHF) show that passing from the $\mathrm{C}_{24}$ fragment to the $\mathrm{C}_{54}$ fragment does not alter the interaction energy any more. The next three curves show the interaction energy for the three metals on the $\mathrm{C}_{24}$ fragment, as calculated with the RSHLDA scheme. The last two lines show that the potential surface, calculated at the RSHLDA level for Zn, is flat over the different possible adsorption positions. 
<smiles>c1cc2ccc3ccc4ccc5ccc6ccc1c1c2c3c4c5c61</smiles>

a)

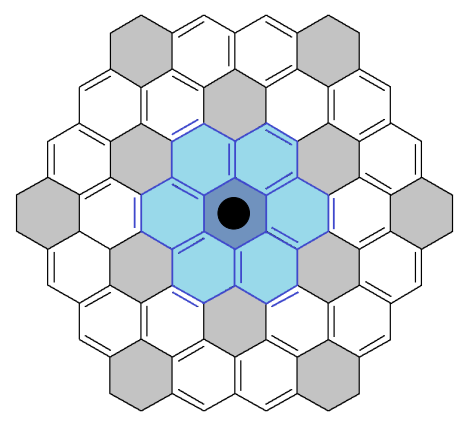

b)

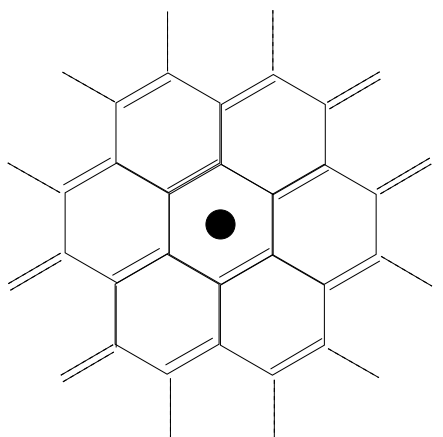

c)

FIG. 2. The alternation of single and double bonds as obtained from the Boys localization of the $\mathrm{C}_{24}$ fragment (upper left panel, a). The upper right panel (b) shows the possible extension to larger fragments, based on the direct continuation of the Boys pattern. Superposition of three shifted structures gives the graphene sheet with equivalent 6-rings. The lower panel (c) shows the alternative set-up with equivalent hexagons, as used in section III D 2. 

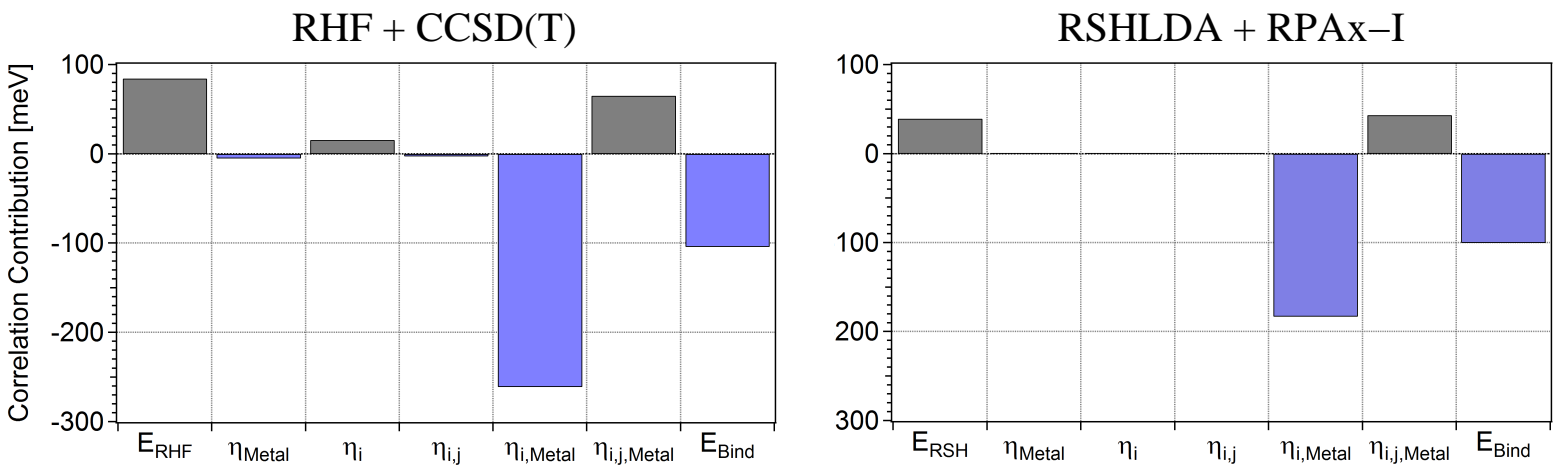

FIG. 3. Contributions of the different classes of increments to the total interactions energy (rightmost item in both diagrams), for $\mathrm{Zn}$ on the $\mathrm{C}_{24}$ at $3.5 \AA$. For the $\mathrm{RPAx}-\mathrm{I}$ increments the dispersion approximation was applied, thus there are no intra-fragment increments at all recorded, only inter-fragment contributions are accounted for. 


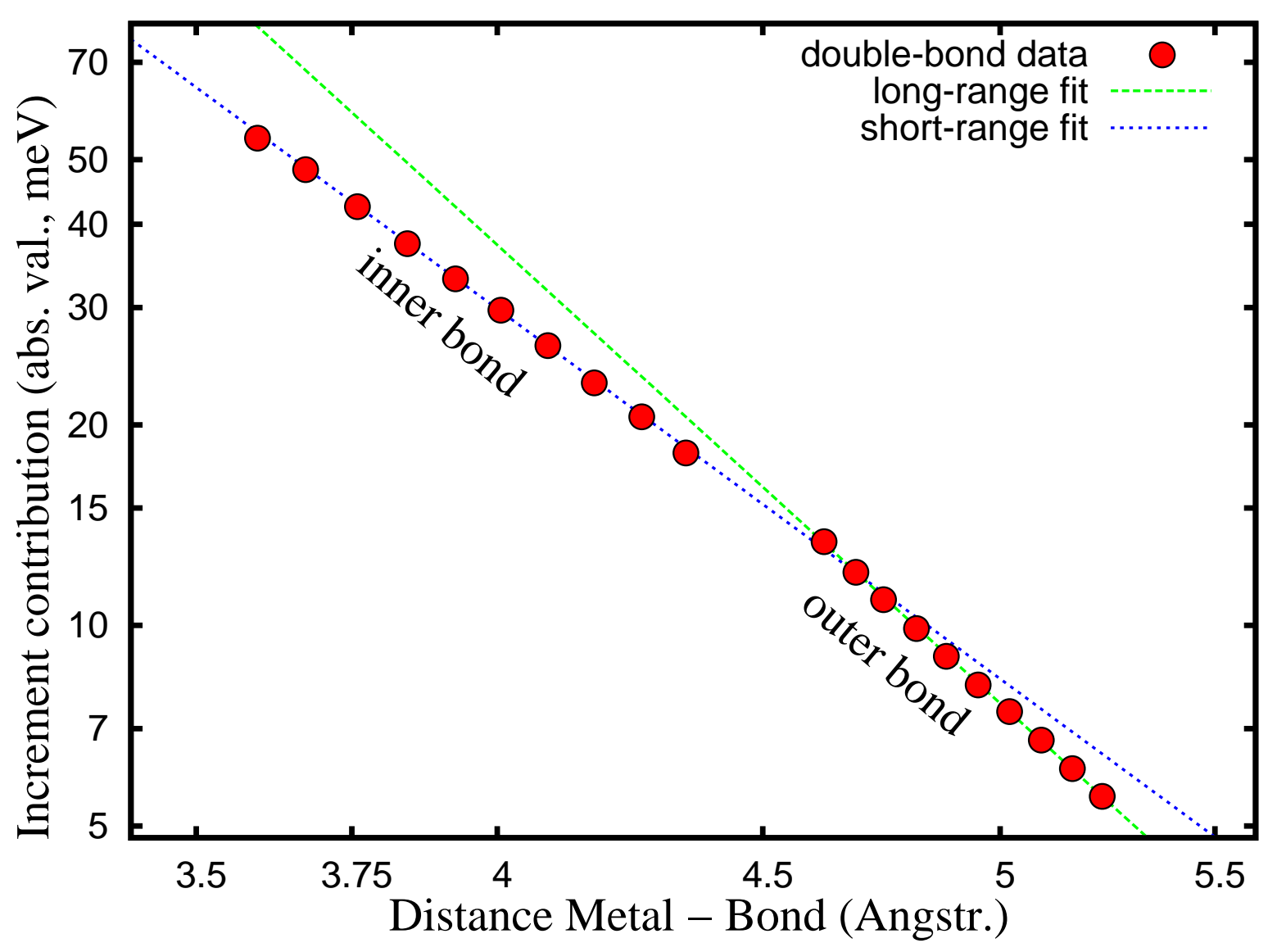

FIG. 4. The two-segment fit in a log-log plot, at the hand of the double bonds of the $\mathrm{Zn} / \mathrm{C}_{24}$ system. 


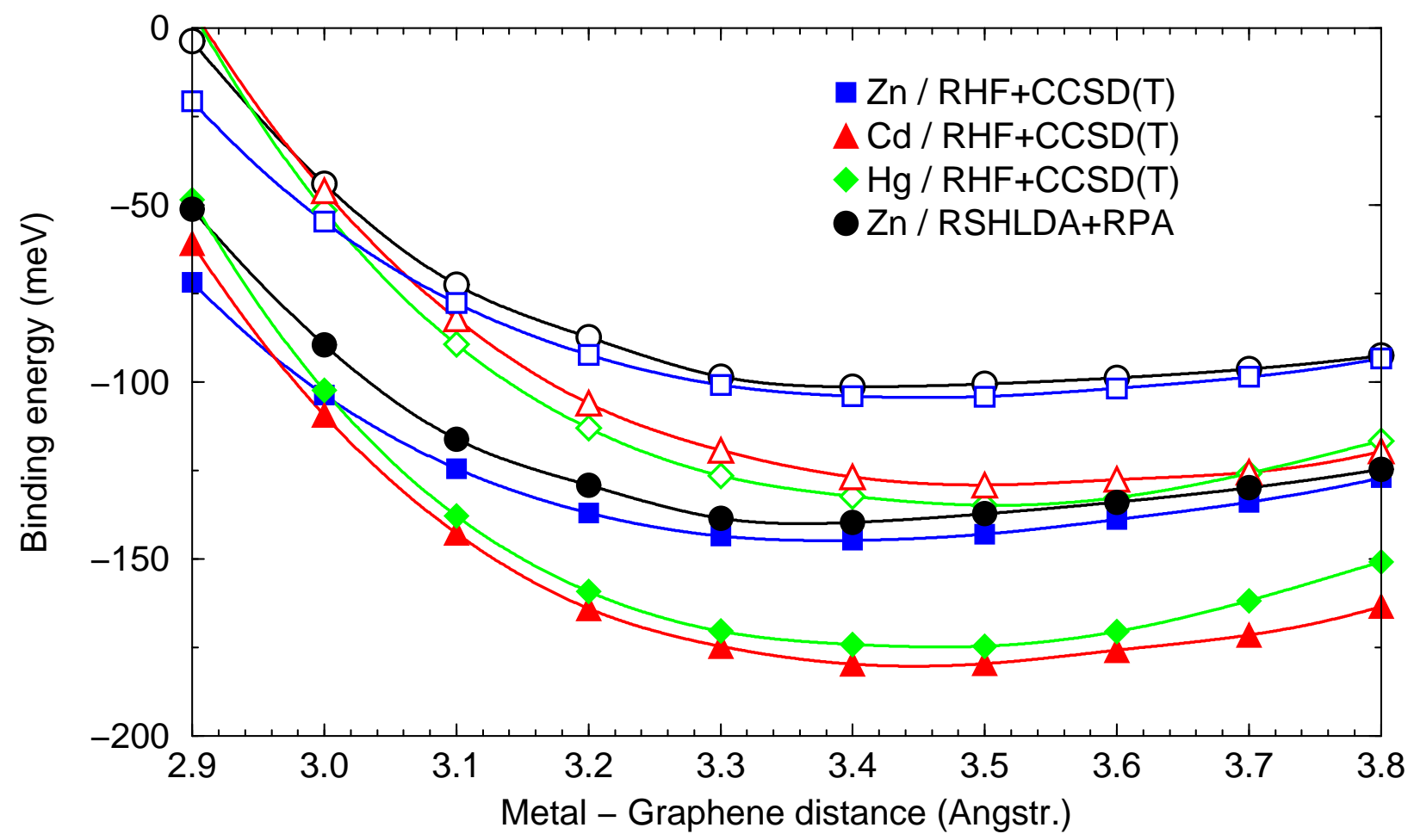

FIG. 5. Result of the extrapolation for the total binding energies in the hollow position of $\mathrm{Zn}$ (RSH+RPA and $\mathrm{RHF}+\mathrm{CCSD}(\mathrm{T})), \mathrm{Cd}$ and $\mathrm{Hg}(\mathrm{RHF}+\mathrm{CCSD}(\mathrm{T}))$. The hollow symbols stand for the results on the $\mathrm{C}_{24}$ fragment, and full symbols give the corresponding extrapolated data. 


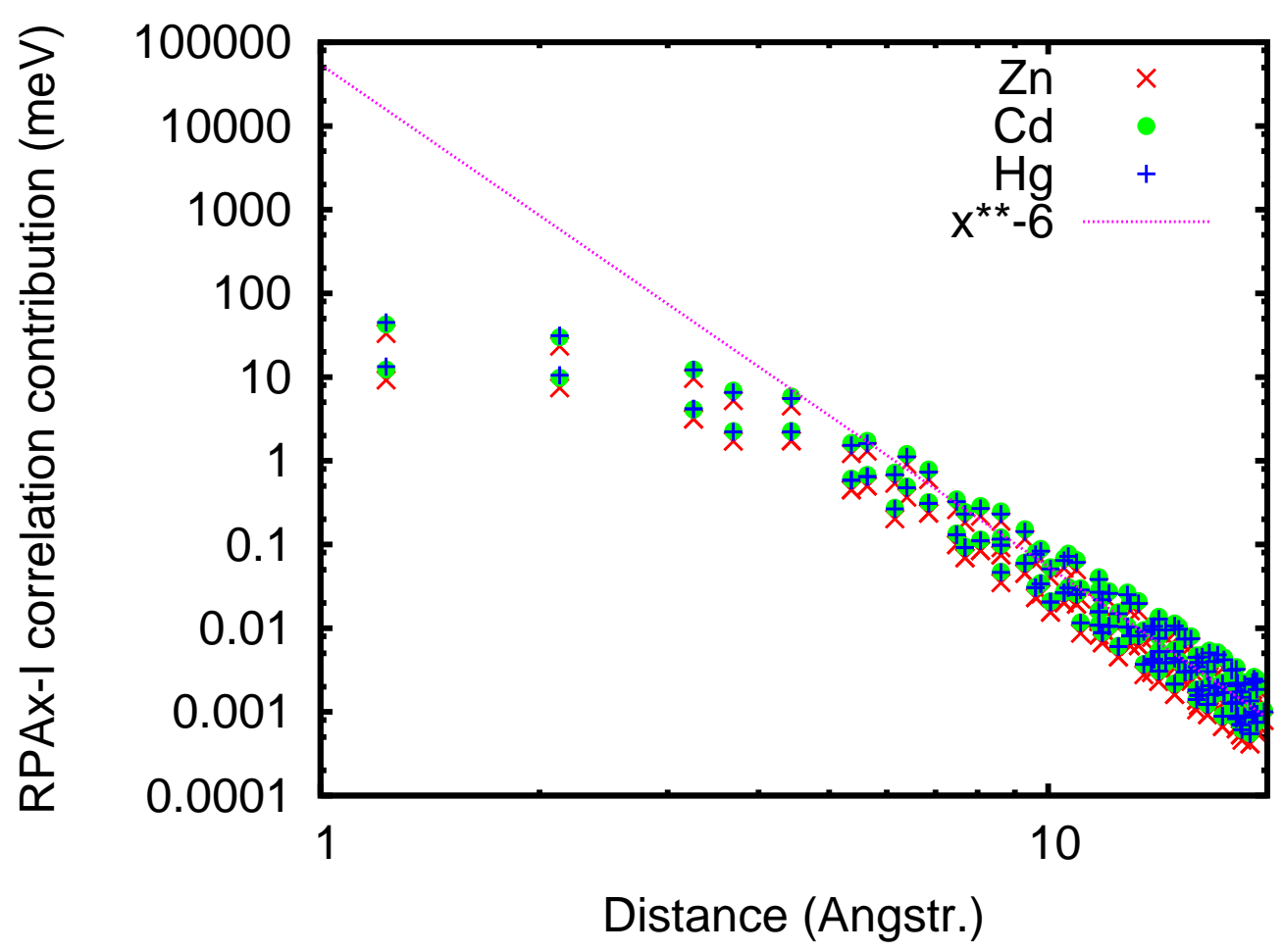

FIG. 6. The individual contributions in a double-logarithmic plot for a single metal-graphene distance of $3.0 \AA$, for showing easily the $1 / R^{6}$ asymptotic behavior. For the short distances we see the two different levels of contributions of 2 bonds in the double-bond case and one bond for the single-bond increments, about three times less important. All three metals yield about the same graph. The $1 / R^{6}$ curve is scaled with 2 a.u. $\times \AA^{6}$. 


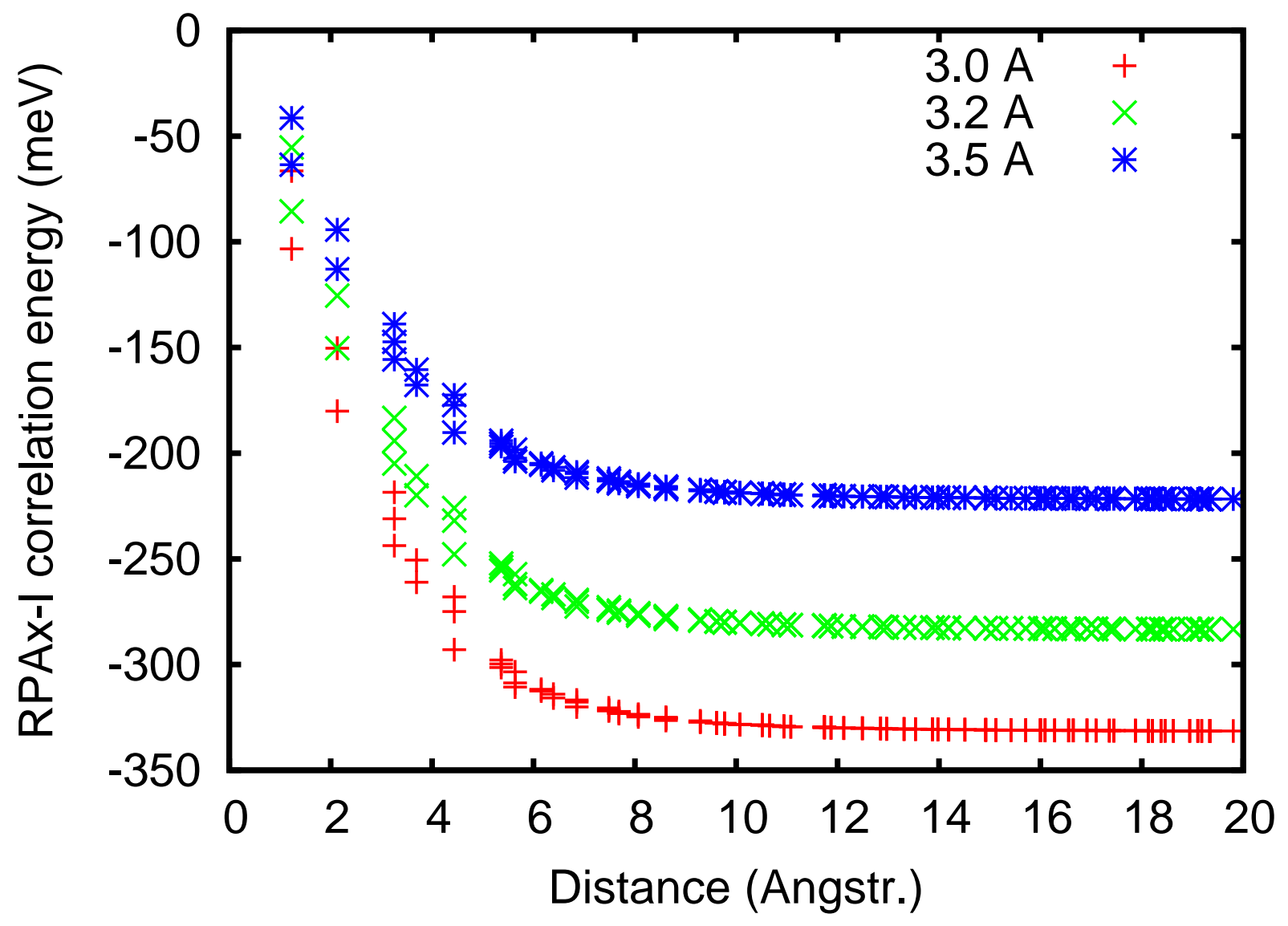

FIG. 7. The cumulative calculation of the correlation contribution to the binding energy, for the $\mathrm{Zn}$ atom adsorption at the hollow position, now at three different Zn-graphene distances. 